\title{
Bondage Number of a Butterfly Graph
}

\author{
${ }^{1}$ Dr . P.Vijaya Jyothi ${ }^{2}$ Dr Indrani Pramod Kelkar \\ ${ }^{I}$ Assistant Proffesor in Mathematics, Dept of S\&H, N.B.K.R.I.T, Vidhyanar ,SPSN, Andhra Pradesh, India, \\ ${ }^{2}$ Assistant Vice President, Academics, GATEFORUM, Hyderabad,
}

\begin{abstract}
Domination Theory is an important branch of Graph Theory that has wide range of applications to various branches of Science and Technology. A new family of graphs called Butterfly Graphs is introduced and study of its parameters is under progress. Butterfly Graphs are undirected graphs and are widely used in interconnection networks.

The bondage number $\mathrm{b}(\mathrm{G})$ of a graph $\mathrm{G}$ is the minimum cardinality among all sets $\mathrm{S} \subset \mathrm{E}(\mathrm{G})$ such that $\gamma(\mathrm{G}$ S) $>\gamma(\mathrm{G})$.

In this paper bondage numbers of butterfly graph are studied for various values of $n=2,3,4$, then bondage number of $\mathrm{BF}(\mathrm{n})$ is generalized.
\end{abstract}

Keywords: Butterfly graph, Domination set, bondage number Subject Classification: 68R10

\section{INTRODUCTION}

A concept connected to domination numbers, called bondage number of a graph was studied by Fink, Jacobson, Kinch and Roberts [5]. The bondage number $b(G)$ of a graph $\mathrm{G}$ is the minimum cardinality among all sets $\mathrm{S} \subset$ $\mathrm{E}(\mathrm{G})$ such that $\gamma(\mathrm{G}-\mathrm{S})>\gamma(\mathrm{G})$.

Thus the bondage number of $\mathrm{G}$ is the minimum cardinality among all subsets of edges whose removal will render every minimum dominating set in $\mathrm{G}$ a non-dominating set in the resultant spanning subgraph. Since the domination number of every spanning subgraph of a non empty graph $\mathrm{G}$ is at least $\gamma(\mathrm{G})$, the bondage number is well defined.

In this chapter various bondage numbers of butterfly graph are studied. Further bondage number and some bounds on them are discussed.

For results on dominating sets and domination number of $\mathrm{BF}(\mathrm{N})$ required to study bondage number we refer Chapters 4,5 of $[8]$.

The cases $\mathrm{n}=2,3,4$ are considered separately and then the result is generalized by using Recursive Construction 2 for all values of $n>4$.

\section{BONDAGE NUMBER OF A BUTTERFLY GRAPH}

Lemma 1 : The bondage number of $\mathrm{BF}(2)$ is 2 .

Proof : Consider the butterfly graph BF(2). The domination number of BF (2) is 2 ( Lemma 4.5, Chapter 4 of [8] ). In $\mathrm{BF}(2)$ every vertex in one level is adjacent to 3 vertices of the other level. So by the definition of edges in $\operatorname{BF}(2)$, observe that a vertex $(0 ; s)$ of $\mathrm{L}_{0}$ is adjacent to a vertex $(0 ; \mathrm{r})$ of $\mathrm{L}_{1}$, for $\mathrm{s}, \mathrm{r}=0,1,2,3$, where $\mathrm{s}+\mathrm{r} \neq 3$. Let $\mathrm{e}$ be an edge joining the vertices $(0 ; \mathrm{s})$ and $(0 ; \mathrm{r})$ such that $\mathrm{s}+\mathrm{r} \neq 3$. Consider the graph $\mathrm{BF}(2) \backslash\{\mathrm{e}\}$. Let $\mathrm{D}=\left\{\left(0 ; \mathrm{m}_{1}\right),\left(1 ; \mathrm{m}_{2}\right) / \mathrm{m}_{1}+\mathrm{m}_{2}=3\right\}$. The four possibilities for $\mathrm{D}$ are given below.
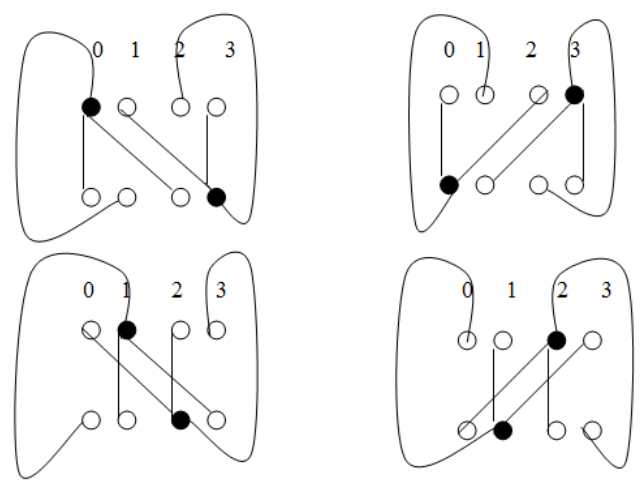

Fig. 1 BF(2) 
From the above possibilities, it can be verified that $\mathrm{D}$ given above also becomes a dominating $\operatorname{set}$ of $\mathrm{BF}(2) \backslash$ e of cardinality 2 for any selection of edge e. Therefore $\gamma(\mathrm{BF}(2) \backslash \mathrm{e})=2=\gamma(\mathrm{BF}(2))$. That is , deletion of a single edge from $\mathrm{BF}(2)$ does not alter the domination number. Now the deletion of two edges from $\mathrm{BF}(2)$ increases the domination number is shown below.

Let $e_{1}, e_{2}$ be any two winged edges of $B F(2)$, given by $e_{1}=\{(o ; r),(1 ; s)\}, e_{2}=\left\{\left(o ; t_{1}\right),\left(1 ; t_{2}\right)\right\}$, where $s+r$ $\neq 3, t_{1}+t_{2} \neq 3$. Let $F=\left\{e_{1}, e_{2}\right\}$. Consider the graph $B F(2) \backslash F$. Without loss of generality take $r=0, s=1$ and $t_{1}=3$ and $t_{2}=2$. Then $e_{1}=\{(0 ; 0),(1 ; 1)\}, e_{2}=\{(0 ; 3),(1 ; 2)\}$. Then for all possible choices of $D$ given above, it can be verified that no $\mathrm{D}$ can dominate one of the end vertices of either $\mathrm{e}_{1}$ or $\mathrm{e}_{2}$.

Consider the dominating set $\mathrm{D}=\{(0 ; 0),(1 ; 3)\}$. It is obvious that, this set can not dominate the vertex $(1,1)$ , as $\mathrm{e}_{1}$ is the only edge joining $(0 ; 0)$ and $(1 ; 1)$. Therefore, adjoin $(1 ; 1)$ to $\mathrm{D}$, so that $\mathrm{D}$ becomes $\mathrm{D}=\{(0$; $0),(1 ; 3),(1 ; 1)\}$ and $\mathrm{D}$ dominates all vertices of $\mathrm{BF}(2) \backslash \mathrm{F}$. Further this set $\mathrm{D}$ is minimum. That is $\gamma(\mathrm{BF}(2) \backslash \mathrm{F})$ $=3$ and hence $\gamma(\mathrm{BF}(2) \backslash \mathrm{F})>\gamma(\mathrm{BF}(2))$.

For all possible values of $r, s$ and $t_{1}, t_{2}$ such that $s+r \neq 3, t_{1}+t_{2} \neq 3$, any of the above mentioned dominating sets can not dominate one of the end vertices of $\mathrm{e}_{1}$ or $\mathrm{e}_{2}$.

Hence for all possible choices of $\mathrm{e}_{1}, \mathrm{e}_{2}, \gamma(\mathrm{BF}(2) \backslash \mathrm{F})>\gamma(\mathrm{BF}(2))$. Therefore $\mathrm{b}(\mathrm{BF}(2))=2$. $\square$

Lemma 2 : The bondage number of $\mathrm{BF}(3)$ is 4.

Proof : Consider the graph $\mathrm{BF}(3)$. It is known that $\gamma(\mathrm{BF}(3))=6$ (Lemma 4.6, Chapter 4 of [8]). Let $\mathrm{F}$ be a set of edges incident on a vertex, say $(1 ; r), r=0,1,2, \ldots 7$. Consider the graph $B F(3) \backslash F$. This vertex becomes isolated in $\mathrm{BF}(3) \backslash \mathrm{F}$. Then $|\mathrm{F}|=4$, since the degree of every vertex in $\mathrm{BF}(\mathrm{n})$ is 4 . It is observed in Chapter 2 that there are six triangles in $\mathrm{BF}(3)$ and each triangle contributes a vertex to a dominating set of $\mathrm{BF}(3)$. Thus to dominate the remaining 11 vertices in the left copy of $\operatorname{BF}(3) \backslash(1 ; r), 3$ vertices are needed and to dominate 12 vertices in the right copy of $\mathrm{BF}(3) \backslash(1 ; \mathrm{r})$, again 3 vertices are needed. Thus these 6 vertices dominate all the vertices of $\mathrm{BF}(3) \backslash(1 ; \mathrm{r})$. So along with the vertex $(1 ; \mathrm{r})$, a dominating set of $\mathrm{BF}(3) \backslash \mathrm{F}$ has minimum cardinality 7 .

Thus $\gamma(\mathrm{BF}(3) \backslash \mathrm{F}) \neq \gamma(\mathrm{BF}(3))$.

Now we claim that removal of any 3 edges from $\mathrm{BF}(3)$ does not increase the domination number of $\mathrm{BF}(3)$. This is proved by taking the edges in $\mathrm{K}_{2,2} \mathrm{~s}$, between $\mathrm{L}_{0}, \mathrm{~L}_{1}$ and $\mathrm{L}_{1}, \mathrm{~L}_{2}$.

Case 1 : Let $\mathrm{F}=\{\{(0 ; \mathrm{r}),(1 ; \mathrm{r})\},\{(0 ; \mathrm{r}),(1 ; \mathrm{r}+2)\},\{(0 ; \mathrm{s}),(1 ; \mathrm{s})\}$ where $\mathrm{r}=0,1$ and $|r-s|=1$. Consider the graph $\mathrm{BF}(3) \backslash \mathrm{F}$. The selection of vertices for domination in $\mathrm{BF}(3) \backslash \mathrm{F}$ is as follows. Let $\mathrm{D}_{1}$ denote a dominating set of $\mathrm{BF}(3) \backslash \mathrm{F}$ in the left copy.

Consider a $K_{2,2}$ between $L_{0}$ and $L_{1}$ incident on four vertices $(0 ; r),(0 ; r+2),(1 ; r),(1 ; r+2)$ in the left copy for $r=0,1$. Since the edge incident on two vertices $(0 ; r),(1 ; r)$ and the edge incident on two vertices $(0 ; r),(1 ; r+$ $2)$ in the first $K_{2,2}$ are deleted, select the vertex $(0 ; r+2)$ or $(1 ; r)$ into $D_{1}$. First select a vertex of $L_{0}$, say $(0$; $r+2$ ) into $D_{1}$. This vertex dominates two vertices $(1 ; r+2),(1 ; r)$ of $L_{1}$ and one vertex $(2 ; r+2)$ of $L_{2}$.

Consider another $K_{2,2}$ between $L_{0}$ and $L_{1}$ incident on four vertices $(0 ; s),(0 ; s+2),(1 ; s),(1 ; s+2)$ in the left copy, where $|r-s|=1$. The edge incident on two vertices $(0 ; \mathrm{s}),(1 ; \mathrm{s})$ in the second $\mathrm{K}_{2,2}$ is deleted. So select the vertex $(0 ; s+2)$ or $(1 ; s+2)$ into $D_{1}$. As a vertex from $L_{0}$ is already taken into $D_{1}$, we select $(1 ; s+$ 2 ) into $D_{1}$. This vertex dominates two vertices $(0 ; s+2),(0 ; s)$ of $L_{0}$ and one vertex $(2 ; s+2)$ of $L_{2}$

Then $\mathrm{D}_{1}=\{(0 ; \mathrm{r}+2),(1 ; \mathrm{s}+2)\}=\left\{\left(0 ; \mathrm{r}_{1}\right),\left(1 ; \mathrm{s}_{1}\right)\right\}$, say. That is $\mathrm{r}+2=\mathrm{r}_{1}$ and $\mathrm{s}+2=\mathrm{s}_{1}$. Now select a vertex from $\mathrm{L}_{2}$ into $\mathrm{D}_{1}$. Then select the vertex $\left(2 ; \mathrm{t}_{1}\right)$ in $\mathrm{L}_{2}$ such that $\left|t_{1}-s_{1}\right|=2$ or 1 . Thus $\mathrm{D}_{1}=\left\{\left(0 ; \mathrm{r}_{1}\right),\left(1 ; \mathrm{s}_{1}\right)\right.$, $\left.\left(2 ; \mathrm{t}_{1}\right)\right\}$.

For the case $r=0, s=1$, we get $D_{1}=\left\{(0 ; 2),(1 ; 3),\left(2 ; t_{1}\right)\right\}$ where $\left|t_{1}-3\right|=2$ or 1 . We take $t_{1}=1$. So $D_{1}$ $=\{(0 ; 2),(1 ; 3),(2 ; 1)\}$.

The following figure illustrates the selection of vertices into $\mathrm{D}_{1}$.

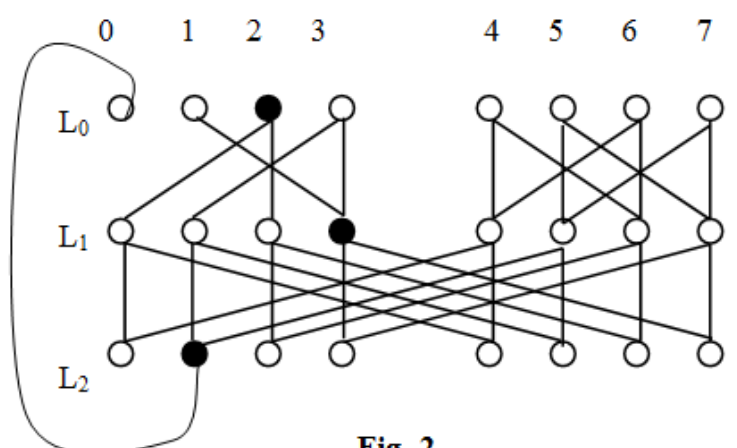

Fig. 2 
From the figure, observe that the vertices in $D_{1}$, dominate all the vertices except $(2 ; r)=(2 ; 0)$ in the left copy of $\mathrm{BF}(3) \backslash \mathrm{F}$.

The mirror image of $\mathrm{D}_{1}$ in the right copy, denoted by $\mathrm{D}_{2} \quad$ contains the vertices $\left\{\left(0 ; \mathrm{r}_{2}\right),\left(1 ; \mathrm{s}_{2}\right),\left(2 ; \mathrm{t}_{2}\right)\right\}$ where $r_{1}+r_{2}=7, \quad s_{1}+s_{2}=7, \quad t_{1}+t_{2}=7$. Then as per the above values of $r_{1}, s_{1}, t_{1}, D_{2}=\{(0 ; 5),(1 ; 4)$, $(2 ; 6)\}$.

The following figure illustrates the selection of vertices into $\mathrm{D}_{2}$.

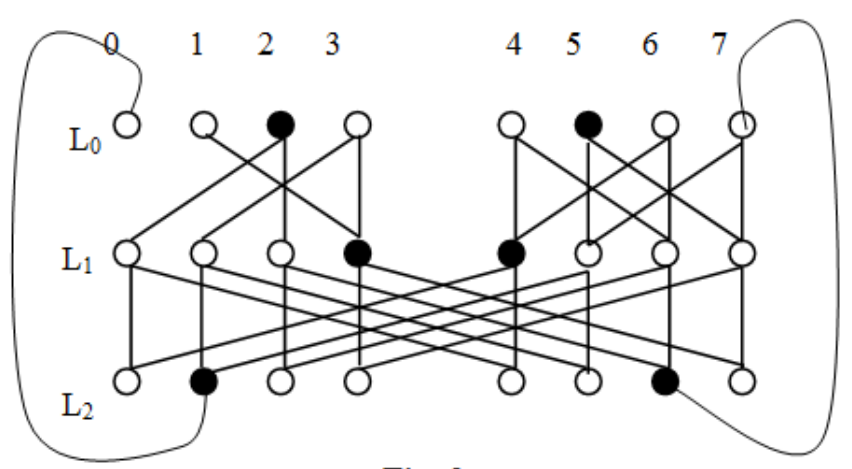

Fig. 3

It can be verified from the figure that $\mathrm{D}_{2}$ dominates all the vertices in the right copy of $\mathrm{BF}(3) \backslash \mathrm{F}$ except the vertex $\left(2 ; \mathrm{t}_{2}+1\right)=(2 ; 7)$.

Now the undominated vertex $(2 ; \mathrm{r})=(2 ; 0)$ in the left copy is dominated by the selected vertex $(1 ; 4)=(1$; $\left.\mathrm{s}_{2}\right)$ and the undominated vertex $(2 ; 7)=\left(2 ; \mathrm{t}_{2}+1\right)$ in the right copy is dominated by the selected vertex $(1 ; 3)$ $=\left(1 ; \mathrm{s}_{1}\right)$.

Let $\mathrm{D}=\mathrm{D}_{1} \cup \mathrm{D}_{2}$. Then all vertices of $\mathrm{BF}(3) \backslash \mathrm{F}$ are dominated by the 6 selected vertices in $\mathrm{D}$. Therefore $\gamma$ $(\mathrm{BF}(3))=\gamma(\mathrm{BF}(3) \backslash \mathrm{F})$.

For other choices of edges in these two $\mathrm{K}_{2,2} \mathrm{~s}$, it can be shown that the domination number for $\mathrm{BF}(3)$ and $\mathrm{BF}(3) \backslash \mathrm{F}$ is unaltered. Similar is the case if $\mathrm{K}_{2,2} \mathrm{~s}$ are taken between $\mathrm{L}_{0}$ and $\mathrm{L}_{1}$ in the right copy .

Case 2 : Let $F=\left\{\left\{\left(1 ; r^{1}\right),\left(2 ; r^{1}\right)\right\},\left\{\left(1 ; r^{1}\right),\left(2 ; r^{1}+2^{2}\right)\right\},\left\{\left(1 ; s^{1}\right), \quad\left(2 ; s^{1}\right)\right\}\right.$ where $r^{1}=0,1,2,3,\left|r^{1}-s^{1}\right|$ $=1$. Consider the graph $\mathrm{BF}(3) \backslash \mathrm{F}$. The selection of vertices for domination in $\mathrm{BF}(3) \backslash \mathrm{F}$ is as follows. Let $\mathrm{D}_{1}$ denote a dominating set of $\mathrm{BF}(3) \backslash \mathrm{F}$ in the left copy of $\mathrm{BF}(3)$.

Consider a $K_{2,2}$ between $L_{1}$ and $L_{2}$ incident on four vertices $\left(1 ; r^{1}\right),\left(1 ; r^{1}+2^{2}\right),\left(2 ; r^{1}\right),\left(2 ; r^{1}+2^{2}\right)$ for $r^{1}=0$, $1,2,3$. Since the edge incident on two vertices $\left(1 ; r^{1}\right),\left(2 ; r^{1}+2^{2}\right)$ and the edge incident on two vertices $\left(1 ; r^{1}\right)$, $\left(2 ; r^{1}\right)$ in the first $K_{2,2}$ are deleted, select the vertex $\left(1 ; r^{1}+2^{2}\right)$ or $\left(2 ; r^{1}\right)$ into $D_{1}$. First select a vertex of $L_{2}$, say $\left(2 ; r^{1}\right)$ into $D_{1}$. This vertex dominates two vertices $\left(1 ; r^{1}+2^{2}\right)$ of $L_{1}$ and one vertex $\left(0 ; r^{1}\right)$ of $L_{0}$.

Consider another $K_{2,2}$ between $L_{1}$ and $L_{2}$ incident on four vertices $\left(1 ; s^{1}\right),\left(1 ; s^{1}+2^{2}\right),\left(2 ; s^{1}\right),\left(2 ; s^{1}+2^{2}\right)$, where $\left|r^{1}-s^{1}\right|=1$. Again, since the edge incident on two vertices $\left(1 ; s^{1}\right),\left(2 ; s^{1}\right)$ in the second $K_{2,2}$ is deleted, select the vertex $\left(1 ; s^{1}\right)$ or $\left(2 ; s^{1}+2^{2}\right)$ into $D_{1}$. As a vertex from $L_{2}$ is already taken into $D_{1}$, select $(1$; $\left.s^{1}\right)$ of $L_{1}$ into $D_{1}$. This vertex dominates 2 vertices $\left(2 ; s^{1}+2^{2}\right)$ of $L_{2}$ and one vertex $\left(0 ; s^{1}\right)$ of $\mathrm{L}_{0}$. Then $\mathrm{D}_{1}=\left\{\left(2 ; \mathrm{r}^{1}\right),\left(1 ; \mathrm{s}^{1}\right)\right\}$.

Next select a vertex from $L_{0}$ into $D_{1}$. Let this vertex be $\left(0 ; t^{1}\right)$ in $L_{0}$ such that $\left|t^{1}-s^{1}\right|=1$. Thus $D_{1}=\{(2$; $\left.\left.\mathrm{r}^{1}\right),\left(1 ; \mathrm{s}^{1}\right),\left(0 ; \mathrm{t}^{1}\right)\right\}$

For the values of $\mathrm{r}^{1}=0, \mathrm{~s}^{1}=1, \mathrm{D}_{1}=\left\{(2 ; 0),(1 ; 1),\left(0 ; \mathrm{t}^{1}\right)\right\}$ where $\left|t^{1}-s^{1}\right|=1$. So $\mathrm{t}^{1}=2$ and $\mathrm{D}_{1}=\{(2$; $0),(1 ; 1),(0 ; 2)\}$.

The following figure illustrates the selection of vertices into $\mathrm{D}_{1}$. 


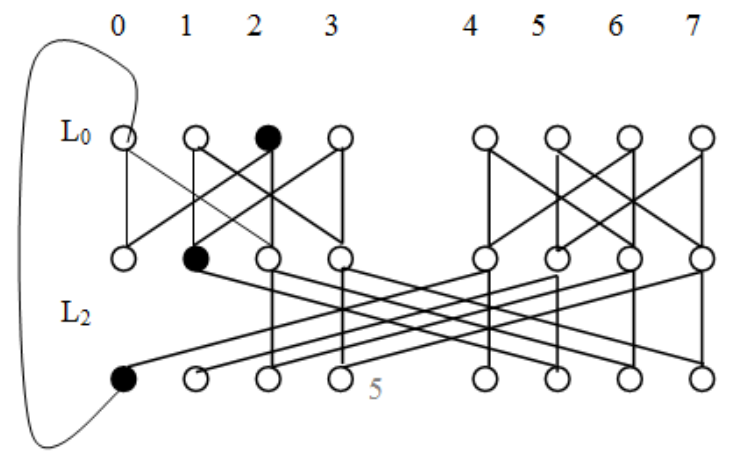

Fig. 4

From the figure it can be verified that the vertices in $D_{1}$, dominate all the vertices except $\left(0 ; t^{1}+1\right)=(1 ; 3)$ in the left copy of $\mathrm{BF}$ (3) $\backslash \mathrm{F}$. As in Case 1 , the mirror image of $\mathrm{D}_{1}$ in the right copy, denoted by $\mathrm{D}_{2}$ contains the vertices $\left\{\left(2 ; \mathrm{r}_{1}{ }^{1}\right),\left(1 ; \mathrm{s}_{1}{ }^{1}\right), \quad\left(0 ; \mathrm{t}_{1}{ }^{1}\right)\right\}$ where $\mathrm{r}^{1}+\mathrm{r}_{1}{ }^{1}=7, \mathrm{~s}^{1}+\mathrm{s}_{1}{ }^{1}=7, \mathrm{t}^{1}+\mathrm{t}_{1}{ }^{1}=7$. Then as per the above values of $\mathrm{r}^{1}, \mathrm{~s}^{1}, \mathrm{t}^{1}, \mathrm{D}_{2}$ becomes $\{(2 ; 7),(1 ; 6)$, $(0 ; 5)\}$.

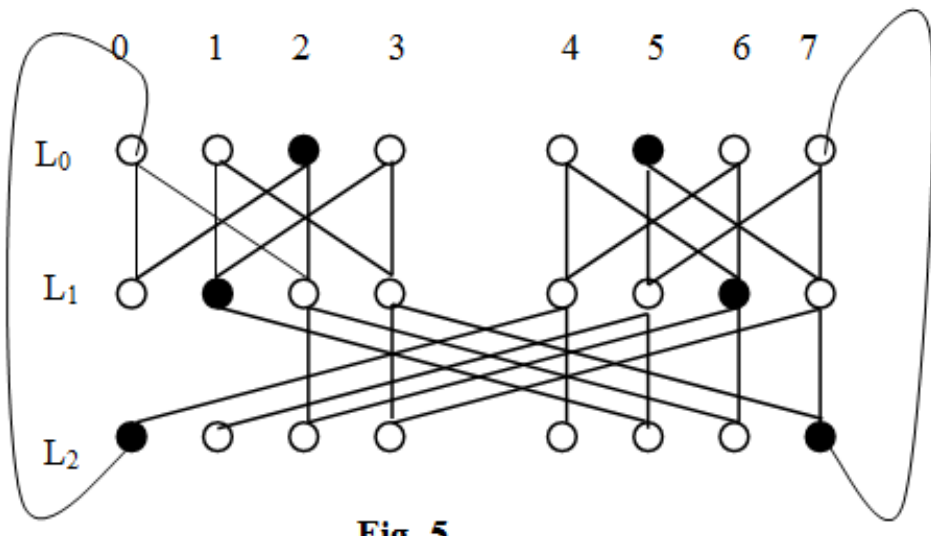

Fig. 5

Again it can be verified from the figure that $\mathrm{D}_{2}$ dominates all the vertices in the right copy of $\mathrm{BF}(3) \backslash \mathrm{F}$ except the vertex $\left(1 ; \mathrm{s}_{1}{ }^{1}+3\right)=(1 ; 4)$. Now the undominated vertex $(1 ; 3)$ in the left copy is dominated by the selected vertex $(2 ; 7)$ and the undominated vertex $(1 ; 4)$ in the right copy is dominated by the selected vertex $(2 ; 0)$. Let $\mathrm{D}=\mathrm{D}_{1} \cup \mathrm{D}_{2}$. Thus all vertices of $\mathrm{BF}(3) \backslash \mathrm{F}$ are dominated by the 6 selected vertices in $\mathrm{D}$. Therefore $\gamma$ $(\mathrm{BF}(3))=\gamma(\mathrm{BF}(3) \backslash \mathrm{F})$.

For other choices of edges in these two $\mathrm{K}_{2,2} \mathrm{~s}$, for $\mathrm{r}^{1}=4,5,6,7$, it can be shown that the domination number of $\mathrm{BF}(3)$ and $\mathrm{BF}(3) \backslash \mathrm{F}$ is unaltered.

Similarly for any choice of three edges in $\mathrm{BF}(3)$ between $\mathrm{L}_{2}$ and $\mathrm{L}_{0}$, it can be shown that the domination number of $\mathrm{BF}(3)$ and $\mathrm{BF}(3) \backslash \mathrm{F}$ is unaltered.

Thus $\mathrm{b}(\mathrm{BF}(3))=4$.

Lemma 3 : The bondage number of $\mathrm{BF}(4)$ is 4 .

Proof : Consider the graph $\mathrm{BF}(4)$. Then $\gamma(\mathrm{BF}(4))=16$ (Lemma 4.7, Chapter 4 of [8]). Consider a set $\mathrm{F}=$ $\left\{\mathrm{e}_{1}, \mathrm{e}_{2}, \mathrm{e}_{3}, \mathrm{e}_{4}\right\}$ of edges in $\mathrm{BF}(4)$. Suppose these 4 edges in $\mathrm{F}$ are incident on a single vertex say $(\mathrm{k} ; \mathrm{m})$. Then removal of these 4 edges from $\mathrm{BF}(4)$ makes $(\mathrm{k} ; \mathrm{m})$ isolated in $\mathrm{BF}(4) \backslash \mathrm{F}$. Hence this vertex is to be included into every dominating set of $\mathrm{BF}(4) \backslash \mathrm{F}$.

Let $\mathrm{D}_{1}$ denote a dominating set of $\mathrm{BF}(4)$. From Recursive Construction 1, we know that $\mathrm{BF}(4)$ has two copies of $\mathrm{BF}(3)$ and a level $\mathrm{L}_{4}$ with 16 vertices. Each of this copy includes 8 vertices into any dominating set of $\mathrm{BF}(4)$. Suppose $(\mathrm{k} ; \mathrm{m}$ ) belongs to $\mathrm{BF}(3)$ in the left copy of $\mathrm{BF}(4)$.

Now to dominate the remaining vertices of the left copy of $\mathrm{BF}(4)$, it is must to include 8 vertices into $\mathrm{D}_{1}$. Also 8 vertices from the right copy of $\mathrm{BF}(4)$ are to be included into $\mathrm{D}_{1}$. Now these 16 vertices in $\mathrm{D}_{1}$ will dominate all the vertices of $\mathrm{BF}(4)$, except $(\mathrm{k} ; \mathrm{m})$, since it is isolated.

Thus cardinality of a dominating set of $\mathrm{BF}(4) \backslash \mathrm{F}$ becomes $16+1=17$. This is the minimum cardinality, as any set of cardinality 16 cannot dominate $\mathrm{BF}(4) \backslash \mathrm{F}$.

Thus $\gamma(\mathrm{BF}(4) \backslash \mathrm{F})>\gamma(\mathrm{BF}(4))$. 
Hence $\mathrm{b}(\mathrm{BF}(4)) \geq 4$.

Now we claim that removal of any 3 edges from $\mathrm{BF}(4)$ does not increase the domination number of $\mathrm{BF}(4)$. This is proved by taking the edges in $\mathrm{K}_{2,2} \mathrm{~s}$, between $\mathrm{L}_{0}, \mathrm{~L}_{1}$ and $\mathrm{L}_{1}, \mathrm{~L}_{2}$.

Case 1 : Let $\mathrm{F}=\{\{(0 ; \mathrm{r}),(1 ; \mathrm{r})\},\{(0 ; \mathrm{s}),(1 ; \mathrm{s})\},\{(0 ; \mathrm{s}), \quad(0 ; \mathrm{s}+2)\}$, where $\mathrm{r}=0,1, \quad|r-s|=1$.

Consider the graph $\mathrm{BF}(4) \backslash \mathrm{F}$. The selection of vertices for domination in $\mathrm{BF}(4) \backslash \mathrm{F}$ is as follows. Let $\mathrm{D}_{1}$ denote a dominating set of $\mathrm{BF}(4) \backslash \mathrm{F}$.

Consider a $\mathrm{K}_{2,2}$ between $\mathrm{L}_{0}$ and $\mathrm{L}_{1}$ incident on four vertices $(0 ; \mathrm{r}),(0 ; r+2),(1 ; r),(1 ; \mathrm{r}+2)$ in the left copy where $r=0$, Since the edge incident on vertices $(0 ; r),(1 ; r)$ in the first $K_{2,2}$ is deleted, select the vertex $(0 ; \mathrm{r}+2)$ or $(1 ; \mathrm{r}+2)$ into $\mathrm{D}_{1}$. First select a vertexof $\mathrm{L}_{1}$, say $(1 ; \mathrm{r}+2)$ into $\mathrm{D}_{1}$. This vertex dominates two vertices $(0 ; \mathrm{r}+2),(0 ; \mathrm{r})$ of $\mathrm{L}_{0}$ and one vertex $(2 ; \mathrm{r}+2)$ of $\mathrm{L}_{2}$.

Consider another $\mathrm{K}_{2,2}$ between $\mathrm{L}_{0}$ and $\mathrm{L}_{1}$ incident on four vertices $(0 ; \mathrm{s}),(0 ; \mathrm{s}+2),(1 ; \mathrm{s}),(1 ; \mathrm{s}+2)$ in the left copy, where $|r-s|=1$. Again the edge incident on two vertices $(0 ; \mathrm{s}),(1 ; \mathrm{s})$ and the edge incident on two vertices $(1 ; s),(0 ; s+2)$ in the second $K_{2,2}$ are deleted, either the vertex $(1 ; s+2)$ or $(0 ; s+2)$ is to be selected into $D_{1}$. Select the vertex $(1 ; s+2)$ into $D_{1}$. This vertex dominates two vertices $(0 ; s+2),(0 ; s)$ of $\mathrm{L}_{0}$ and one vertex $(2 ; \mathrm{s}+2)$ of $\mathrm{L}_{2}$

Then $\mathrm{D}_{1}=\{(1 ; \mathrm{r}+2),(1 ; \mathrm{s}+2)\}$.

It is observed that there are four $\mathrm{K}_{2,2} \mathrm{~s}$ between $\mathrm{L}_{0}$ and $\mathrm{L}_{1}$ in the left copy of $\mathrm{BF}(4)$. As the next $\mathrm{K}_{2,2} \mathrm{~s}$ are mirror image of the first $K_{2,2} \mathrm{~s}$ in the left copy, we select the mirror image of $D_{1}$ in these $K_{2,2} \mathrm{~s}$. So $D_{1}$ becomes $\mathrm{D}_{1}=\left\{(1 ; \mathrm{r}+2),(1 ; \mathrm{s}+2),\left(1 ; \mathrm{r}_{1}\right),\left(1 ; \mathrm{s}_{1}\right)\right\}$ where $\mathrm{r}_{1}=\mathrm{r}+2^{2}, \mathrm{~s}_{1}=\mathrm{s}+2^{2}$.

Here $\left(1 ; r_{1}\right),\left(1 ; s_{1}\right)$ dominate the vertices $\left(0 ; r_{1}\right),\left(0 ; r_{1}+2\right)$ and $\left(0 ; s_{1}\right),\left(0 ; s_{1}+2\right)$ respectively. Thus all vertices of $\mathrm{L}_{0}$ in the left copy are dominated.

Consider four $\mathrm{K}_{2,2} \mathrm{~s}$ between $\mathrm{L}_{1}$ and $\mathrm{L}_{2}$ incident on vertices $\left\{\left(1 ; \mathrm{r}_{2}\right),\left(1 ; \mathrm{r}_{2}+2^{2}\right),(2 ; \mathrm{r}),\left(2 ; \mathrm{r}_{2}+2^{2}\right)\right\},\{(1$; $\left.\left.\mathrm{s}_{2}\right),\left(1 ; \mathrm{s}_{2}+2^{2}\right),\left(2 ; \mathrm{s}_{2}\right),\left(2 ; \mathrm{s}_{2}+2^{2}\right)\right\},\left\{(1 ; \mathrm{p}),\left(1 ; \mathrm{p}+2^{2}\right),(2 ; \mathrm{p}),\left(2 ; \mathrm{p}+2^{2}\right)\right\},\left\{(1 ; \mathrm{q}),\left(1 ; \mathrm{q}+2^{2}\right),(2 ;\right.$ $\left.\mathrm{q}),\left(2 ; \mathrm{q}+2^{2}\right)\right\}$ respectively, where $r_{2} \neq \mathrm{s}_{2} \neq \mathrm{p} \neq \mathrm{q}$ and $\mathrm{r}_{2}=0,1,2, \ldots .7$ in the left copy of $\mathrm{BF}(4) \backslash$ $\mathrm{F}$. Then select the vertices $\left(2 ; \mathrm{r}_{2}\right),\left(2 ; \mathrm{s}_{2}\right), \quad(2 ; \mathrm{p}),(2 ; \mathrm{q})$ of $\mathrm{L}_{2}$ into $\mathrm{D}_{1}$, as the vertices in $\mathrm{L}_{1}$ are already selected into $\mathrm{D}_{1}$ and any dominating set of $\mathrm{BF}(4) \backslash \mathrm{F}$ contains vertices from two consecutive levels .

Now $\mathrm{D}_{1}=\left\{(1 ; \mathrm{r}+2),(1 ; \mathrm{s}+2),\left(1 ; \mathrm{r}_{1}\right),\left(1 ; \mathrm{s}_{1}\right)\left(2 ; \mathrm{r}_{2}\right),\left(2 ; \mathrm{s}_{2}\right),(2 ; \mathrm{p}),(2 ; \mathrm{q})\right\}$.

Here $\left(2 ; r_{2}\right)$ dominates $\left(1 ; r_{2}\right),\left(1 ; r_{2}+2^{2}\right),\left(2 ; s_{2}\right)$ dominates $\left(1 ; s_{2}\right),\left(1 ; s_{2}+2^{2}\right),(2 ; p)$ dominates $(1 ; p),(1$; $\left.\mathrm{p}+2^{2}\right),(2 ; \mathrm{q})$ dominates $(1 ; \mathrm{q}),\left(1 ; \mathrm{q}+2^{2}\right)$. Thus four vertices of $\mathrm{L}_{1}$ are dominated in the left copy of $B F(4) \backslash F$. Now the selected vertices in $L_{1}$ into $D_{1}$ viz., $(1 ; r+2),(1 ; s+2), \quad\left(1 ; r_{1}\right)=\left(1 ; r+2^{2}\right) \quad(1$; $\left.\mathrm{s}_{1}\right)=\left(1 ; \mathrm{s}+2^{2}\right)$ dominate respectively the vertices $\left(2 ; \mathrm{r}+2+2^{2}\right),\left(2 ; \mathrm{s}+2+2^{2}\right),\left(2 ; \mathrm{r}_{1}\right)=\left(2 ; \mathrm{r}+2^{2}\right), \quad(2$; $\left.\mathrm{s}_{1}\right)=\left(2 ; \mathrm{s}+2^{2}\right)$ of $\mathrm{L}_{2}$. Thus including the selected vertices of $\mathrm{L}_{2}$, all the vertices of $\mathrm{L}_{2}$ in the left copy of $\mathrm{BF}(4) \backslash \mathrm{F}$ are dominated .

The selected vertices in $\mathrm{L}_{2}$ will dominate the vertices $\left(3 ; \mathrm{r}_{2}\right),\left(3 ; \mathrm{s}_{2}\right),(3 ; \mathrm{p}),(3 ; \mathrm{q})$ of $\mathrm{L}_{3}$ in the left copy of $\mathrm{BF}(4) \backslash \mathrm{F}$ and also $\left(3 ; r_{2}+2^{3}\right),\left(3 ; s_{2}+2^{3}\right),\left(3 ; p+2^{3}\right),\left(3 ; q+2^{3}\right)$ of $L_{3}$ in the right copy of $B F(4) \backslash F$. Now the vertices $\left(3 ; r_{2}+2^{2}\right),\left(3 ; s_{2}+2^{2}\right),\left(3 ; p+2^{2}\right), \quad\left(3 ; q+2^{2}\right)$ of $L_{3}$ in the left copy of $B F(4) \backslash F$ are undominated .

For the case $\mathrm{r}=0, \mathrm{~s}=1, \mathrm{r}_{2}=0, \mathrm{~s}_{2}=1, \mathrm{p}=2, \mathrm{q}=3$, we get $\mathrm{D}_{1}=\{(1 ; 2),(1 ; 3),(1 ; 4),(1 ; 5),(2 ; 0),(2 ; 1),(2 ; 2),(2 ; 3)\}$.

The following figure illustrates the selection of vertices into $D_{1}$.

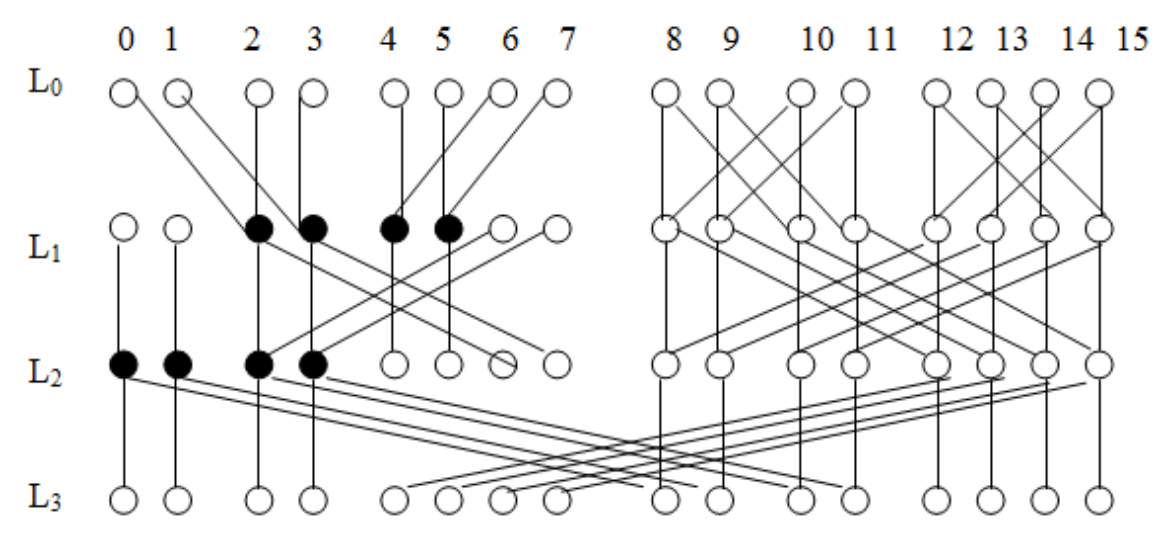

Fig. 6 
From the figure, observe that the vertices in $D_{1}$, dominate all the vertices except $\left(3 ; r_{2}+2^{2}\right)=(3 ; 4),\left(3 ; s_{2}+2^{2}\right.$ )$=(3 ; 5),\left(3 ; p+2^{2}\right)=(3 ; 6),\left(3 ; q+2^{2}\right)=(3 ; 7)$ in the left copy of $B F(4) \backslash F$.

The mirror image of $D_{1}$ in the right copy, denoted by $D_{2}$ contains the vertices $\left\{\left(1 ; r^{1}\right),\left(1 ; s^{1}\right),\left(1 ; r_{1}{ }^{1}\right),\left(1 ; s_{1}{ }^{1}\right)\right.$, $\left.\left(2 ; r_{2}{ }^{1}\right),\left(2 ; s_{2}{ }^{1}\right),\left(2 ; p^{1}\right),\left(2 ; q^{1}\right)\right\}$ where $r+2+r^{1}=15, s+2+s^{1}=15, r_{1}+r_{1}{ }^{1}=15, s_{1}+s_{1}{ }^{1}=15$, $\mathrm{r}_{2}+\mathrm{r}_{2}{ }^{1}=15, \mathrm{~s}_{2}+\mathrm{s}_{2}{ }^{1}=15, \mathrm{p}+\mathrm{p}^{1}=15, \mathrm{q}+\mathrm{q}^{1}=15$. Then as per the above values of $\mathrm{r}, \mathrm{s}, \mathrm{r}_{2}, \mathrm{~s} 2, \mathrm{p}, \mathrm{q}, \mathrm{D}_{2}$ becomes $\{(1 ; 13),(1 ; 12),(1 ; 11),(1 ; 10),(2 ; 15),(2 ; 14),(2 ; 13),(2 ; 12)\}$.

As above it can be shown that the vertices in $\mathrm{D}_{2}$ dominate all the vertices in the right copy of $\mathrm{BF}(4) \backslash \mathrm{F}$ except the vertices $\left(3 ; r_{2}{ }^{1}-7\right),\left(3 ; s_{2}{ }^{1}-5\right),\left(3 ; p^{1}-3\right),\left(3 ; q^{1}-1\right)$ of $L_{3}$ in the right copy of $\quad B F(4) \backslash F$.

The following figure illustrates the selection of vertices into $D_{2}$.

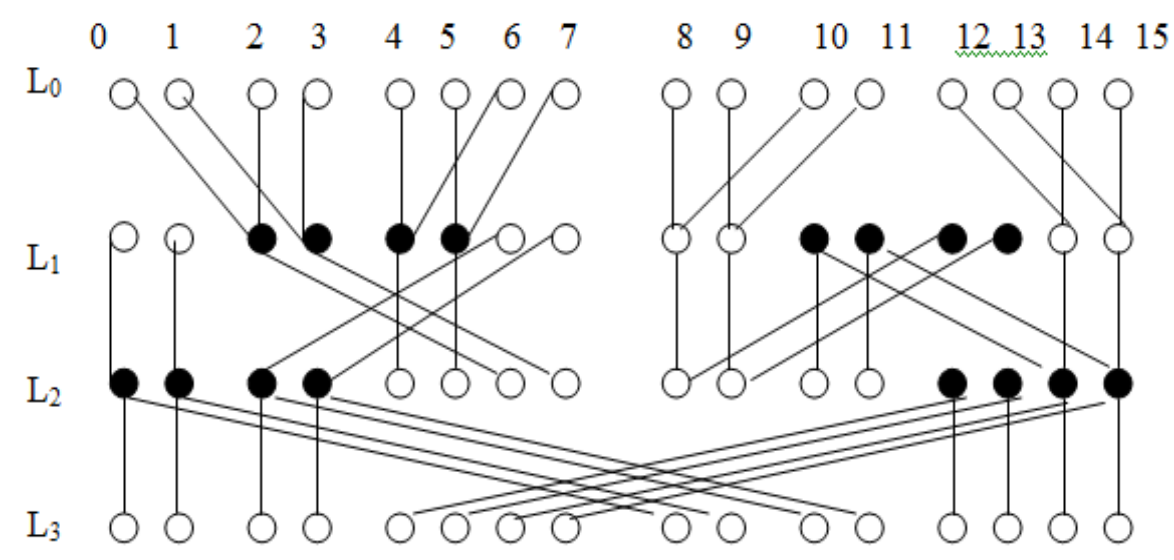

Fig. 7

Again it can be verified from the figure that $\mathrm{D}_{2}$ dominates all the vertices in the right copy of $\mathrm{BF}(4) \backslash \mathrm{F}$ except the vertices $\left(3 ; \mathrm{r}_{2}{ }^{1}-7\right)=(3 ; 8),\left(3 ; \mathrm{s}_{2}{ }^{1}-5\right)=(3 ; 9),\left(3 ; \mathrm{p}^{1}-3\right)=(3 ; 10),\left(3 ; \mathrm{q}^{1}-1\right)=(3 ; 11)$ in the right copy of $\mathrm{BF}(4) \backslash \mathrm{F}$. Now the undominated vertices $(3 ; 4),(3 ; 5),(3 ; 6),(3 ; 7)$ in the left copy are dominated by the selected vertices $(2 ; 12),(2 ; 13),(2 ; 14),(2 ; 15)$ and the undominated vertices $(3 ; 8),(3 ; 9)$, $(3 ; 10),(3 ; 11)$ in the right copy is dominated by the selected vertices $(2 ; 0),(2 ; 1),(2 ; 2),(2 ; 3)$.Let $\mathrm{D}=\mathrm{D}_{1}$ $\cup D_{2}$. Thus all vertices of $B F(4) \backslash F$ are dominated by the 16 selected vertices in D. Therefore $\gamma(B F(4))=\gamma$ $(\mathrm{BF}(3) \backslash \mathrm{F})$.

For other choices of edges in these two $\mathrm{K}_{2,2} \mathrm{~s}$, it can be shown that the domination number for $\mathrm{BF}(4)$ and $B F(4) \backslash F$ is unaltered. Similar is the case if $K_{2,2} s$ are taken between $L_{0}, L_{1}$ and $L_{1}, L_{2}$ in the right copy .

Case 2: Suppose three edges are selected into $F$, between two levels $L_{2}$ and $L_{3}$. Let $F=\{\{(2 ; r),(3 ; r)\},\{$ $(2 ; \mathrm{r}+2),(3 ; \mathrm{r}+2)\},\left\{(2 ; \mathrm{r}),\left(3 ; \mathrm{r}+2^{3}\right)\right\}, \mathrm{r}=0,1,2, \ldots 7$.

As the selection of vertices into a dominating set $\mathrm{D}$ of $\mathrm{BF}(4)$ is from any two consecutive levels, select the vertices of $\mathrm{L}_{2}$ and $\mathrm{L}_{3}$ into $\mathrm{D}$.

Consider the six $\mathrm{K}_{2,2} \mathrm{~s}$ in the left copy of $\mathrm{BF}(4)$ between $\mathrm{L}_{2}$ and $\mathrm{L}_{3}$ given by

$\mathrm{K}_{2,2}=\left\{(2 ; \mathrm{r}),(3 ; \mathrm{r}),\left(2 ; \mathrm{r}+2^{3}\right),\left(3 ; \mathrm{r}+2^{3}\right)\right\}, \mathrm{r}=0,2,4,5,6,7$.

$\mathrm{K}_{2,2}=\left\{(2 ; \mathrm{s}),(3 ; \mathrm{s}),\left(2 ; \mathrm{s}+2^{3}\right),\left(3 ; \mathrm{s}+2^{3}\right)\right\}, \mathrm{s}=\mathrm{r}+2$

$\mathrm{K}_{2,2}=\left\{(2 ; \mathrm{p}),(3 ; \mathrm{p}),\left(2 ; \mathrm{p}+2^{3}\right),\left(3 ; \mathrm{p}+2^{3}\right)\right\}, \mathrm{p}=\mathrm{s}+2$

$\mathrm{K}_{2,2}=\left\{(2 ; \mathrm{q}),(3 ; \mathrm{q}),\left(2 ; \mathrm{q}+2^{3}\right),\left(3 ; \mathrm{q}+2^{3}\right)\right\}, \mathrm{q}=\mathrm{p}+1$

$\mathrm{K}_{2,2}=\left\{(2 ; \mathrm{t}),(3 ; \mathrm{t}),\left(2 ; \mathrm{t}+2^{3}\right),\left(3 ; \mathrm{t}+2^{3}\right)\right\}, \mathrm{t}=\mathrm{q}+1$

$\mathrm{K}_{2,2}=\left\{(2 ; \mathrm{w}),(3 ; \mathrm{w}),\left(2 ; \mathrm{w}+2^{3}\right),\left(3 ; \mathrm{w}+2^{3}\right)\right\}, \mathrm{w}=\mathrm{t}+1$

Let $\mathrm{D}_{1}$ denote a dominating set of $\mathrm{BF}(4) \backslash \mathrm{F}$ in the left copy. Taking into consideration the deletion of edges in $F$, select the following vertices from these $K_{2,2} s$ into $D_{1}$.

Let $D_{1}=\{(3 ; r),(3 ; s),(3 ; p),(2 ; p),(2 ; q),(3 ; t),(2 ; t),(2 ; w)\}$.

Here observe that the vertices in $\mathrm{D}_{1}$ dominate all vertices in the left copy of $\mathrm{BF}(4) \backslash \mathrm{F}$, except the vertices $(2 ; \mathrm{r}$ $+1),(2 ; s+1)$ of $L_{2}$ and $(3 ; r+1),(3 ; s+1)$ of $L_{3}$ For the values of $r=0, s=2, p=4, q=5, t=6, w=7$, $\mathrm{D}_{1}$ becomes $\{(3 ; 0),(3 ; 2),(3 ; 4),(2 ; 4),(2 ; 5),(3 ; 6),(2 ; 6),(2 ; 7)\}$ and the following figure illustrates the selection of vertices into $D_{1}$. 


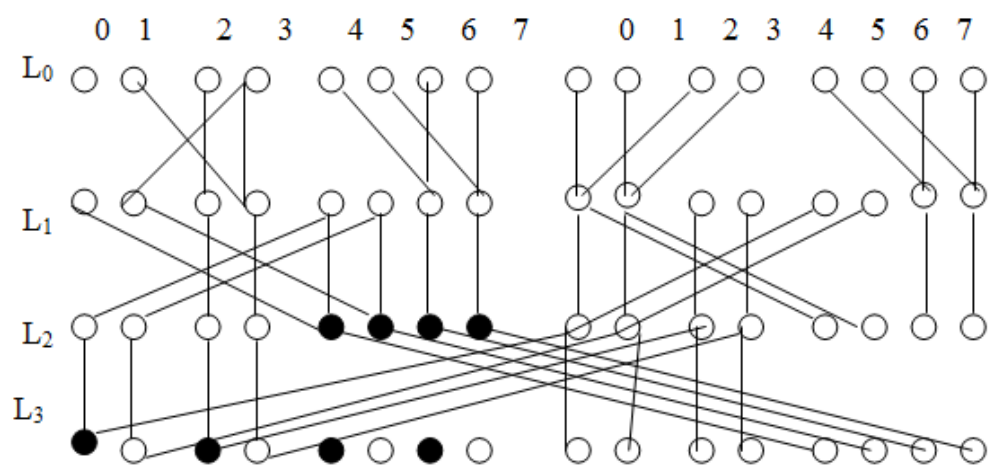

Fig. 8

Here observe that the vertices $(2 ; \mathrm{r}+1)=(2 ; 1),(2 ; \mathrm{s}+1)=(2 ; 3), \quad(3 ; \mathrm{r}+1)=(3 ; 1),(3 ; \mathrm{s}+1)=(3 ; 3)$ are not dominated by the vertices of $\mathrm{D}_{1}$.

Now the vertices in the mirror image of $D_{1}$ denoted by $D_{2}$ are given by $D_{2}=\left\{\left(3 ; r^{1}\right),\left(3 ; s^{1}\right),\left(3 ; p^{1}\right),\left(2 ; p^{1}\right)\right.$, $\left.\left(2 ; \mathrm{q}^{1}\right),\left(3 ; \mathrm{t}^{1}\right),\left(2 ; \mathrm{t}^{1}\right),\left(2 ; \mathrm{w}^{1}\right)\right\}$ where $\mathrm{r}+\mathrm{r}^{1}=15, \mathrm{~s}+\mathrm{s}^{1}=15, \mathrm{p}+\mathrm{p}^{1}=15, \mathrm{q}+\mathrm{q}^{1}=15$, $\mathrm{t}+\mathrm{t}^{1}=15, \mathrm{w}+\mathrm{w}^{1}=15$.

Again it can be shown that the vertices in $\mathrm{D}_{2}$ dominate all vertices in the right copy of $\mathrm{BF}(4) \backslash \mathrm{F}$ except the vertices $\left(2 ; r^{1}-1\right),\left(2 ; s^{1}-1\right)$ of $L_{2}$ and $\left(3 ; r^{1}-1\right)\left(3 ; s^{1}-1\right)$ of $L_{3}$.

For above said values of $\mathrm{r}, \mathrm{s}, \mathrm{p}, \mathrm{q}, \mathrm{t}, \mathrm{w}$, the set $\mathrm{D}_{2}$ becomes $\mathrm{D}_{2}=\{(3 ; 15),(3 ; 13),(3 ; 11),(2 ; 11),(2 ; 10),(3$; $9),(2 ; 9),(2 ; 8) \quad$.Here observe that the vertices $\left(2 ; \mathrm{r}^{1}-1\right)=(2 ; 14)$, $\left(2 ; \mathrm{s}^{1}-1\right)=(2 ; 12),\left(3 ; \mathrm{r}^{1}-1\right)=(3 ; 14),\left(3 ; \mathrm{s}^{1}-1\right)=(3 ; 12)$ are not dominated by the vertices of $\mathrm{D}_{2}$.

The following figure illustrates the selection of vertices into $D_{2}$.

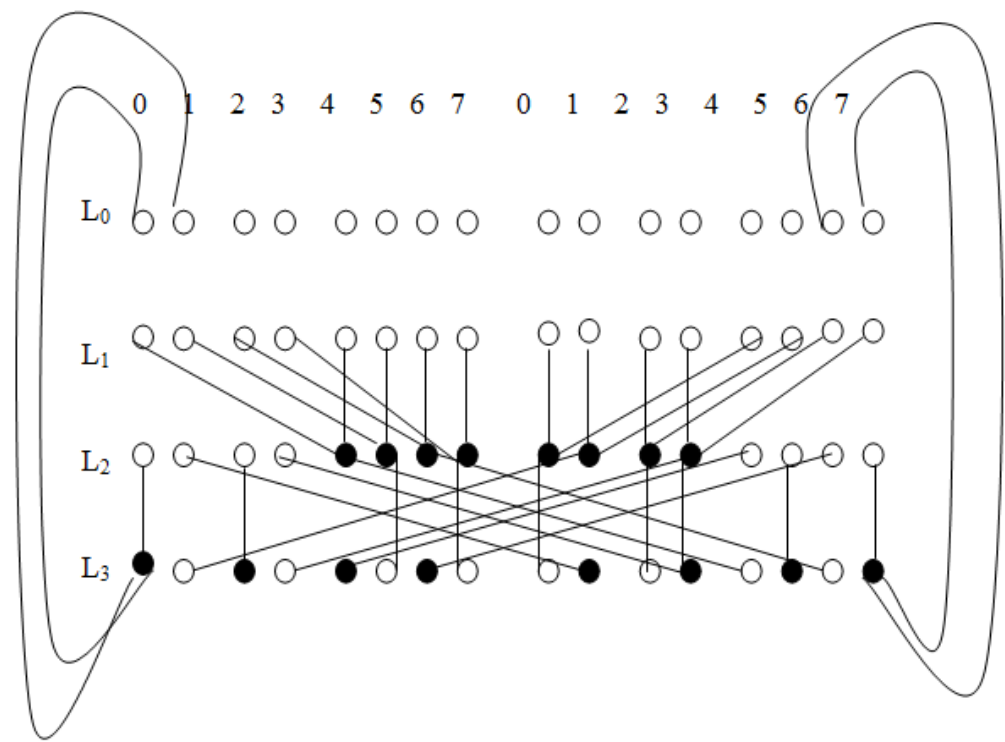

Fig. 9

Now the undominated vertices $(2 ; \mathrm{r}+1)=(2 ; 1), \quad(2 ; \mathrm{s}+1)=(2 ; 3), \quad(3 ; \mathrm{r}+1)=(3 ; 1),(3 ; \mathrm{s}+1)=$ $(3 ; 3)$ in the left copy are dominated by the selected vertices $(3 ; 9),(3 ; 11),(2 ; 9),(2 ; 11)$ respectively. Also the undominated vertices $\left(2 ; \mathrm{r}^{1}-1\right)=(2 ; 14),\left(2 ; \mathrm{s}^{1}-1\right)=(2 ; 12), \quad\left(3 ; \mathrm{r}^{1}-1\right)=(3 ; 14)$, $\left(3 ; \mathrm{s}^{1}-1\right)=(3 ; 12)$ in the right copy are dominated by the selected vertices $(3 ; 6),(3 ; 4),(2 ; 6),(2 ; 4)$ respectively .Let $D=D_{1} \cup D_{2}$. Then all vertices of $B F(4) \backslash F$ are dominated by the 16 selected vertices in D. Therefore $\gamma(\mathrm{BF}(4))=\gamma(\mathrm{BF}(4) \backslash \mathrm{F})$.

For other choices of edges in these two $\mathrm{K}_{2,2} \mathrm{~s}$, it can be shown that the domination number for $\mathrm{BF}(4)$ and $\mathrm{BF}(4) \backslash \mathrm{F}$ is unaltered.

Similarly for any choice of three edges in $\mathrm{BF}(4)$ between $\mathrm{L}_{1}, \mathrm{~L}_{2}$ and $\mathrm{L}_{0}, \mathrm{~L}_{3}$, it can be shown that the domination number of $\mathrm{BF}(4)$ and $\mathrm{BF}(4) \backslash \mathrm{F}$ is unaltered. Thus $\mathrm{b}(\mathrm{BF}(4))=4$. 
Lemma 4 : The bondage number of $\mathrm{BF}(\mathrm{n})$ is

$\mathrm{b}(\mathrm{BF}(\mathrm{n}))=\mathrm{b}(\mathrm{BF}(4))=4$, for $\mathrm{n}>4$.

Proof : From Recursive Construction 1, we know that for $n>4$, every BF(n) has a copy of BF(4) between the first 4 levels $\mathrm{L}_{0}, \mathrm{~L}_{1}, \mathrm{~L}_{2}$ and $\mathrm{L}_{3}$. Removal of 4 edges incident on a single vertex in one of these $\mathrm{BF}(4)$ copies increases the domination number of this copy of $\mathrm{BF}(4)$. Since all these copies are disjoint, this results in the increase of the domination number of $\mathrm{BF}(\mathrm{n})$.

Thus $\mathrm{b}(\mathrm{BF}(\mathrm{n}))=\mathrm{b}(\mathrm{BF}(4))=4$. $\square$

This is illustrated for the graph $\mathrm{BF}(5)$ in the figure given below.

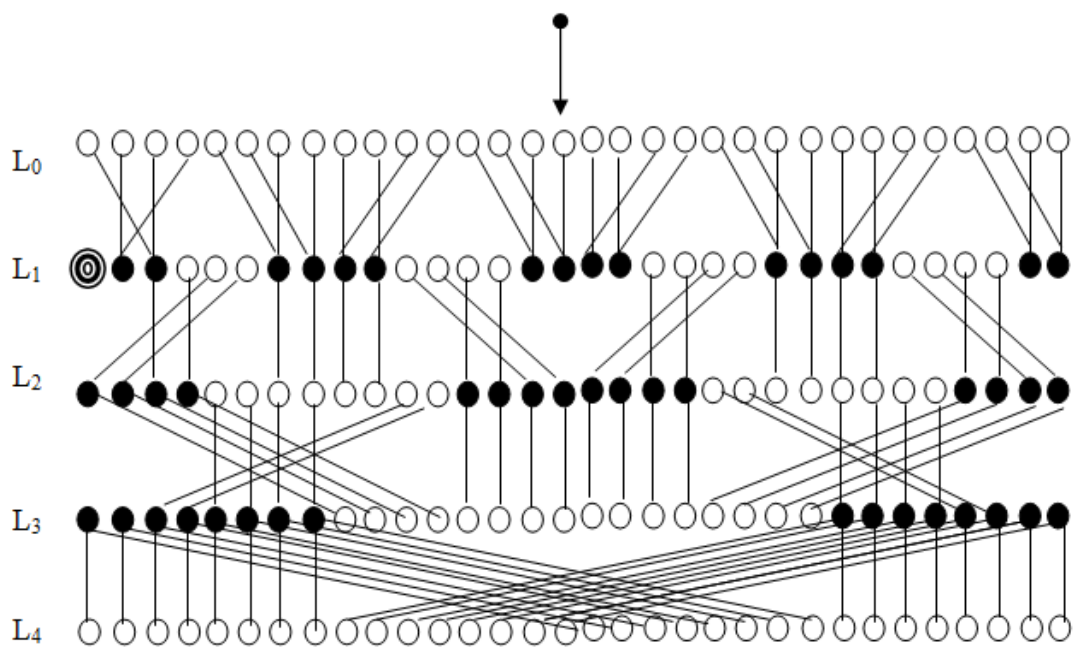

Fig.10 BF(5)

The above results from Lemma 1 through Lemma 4 can be compiled as follows :

Theorem 5 : The bondage number of $\mathrm{BF}(\mathrm{n})$ is

$$
\begin{array}{rlr}
\mathrm{b}(\mathrm{BF}(\mathrm{n})) & =2 & \text { for } \mathrm{n}=2 . \\
& =4 & \text { for } \mathrm{n} \geq 3 . \square
\end{array}
$$

\section{REFERENCES}

[1]. Barth, D., Raspaud, A., Two edge-disjoint Hamiltonian cycles in the Butterfly Graph, Infrom. Process.

[2]. Lett. 51,(1994), 175-179

[3]. Berge, C., Theory of graphs and its Aplications ,Methuen, London(1962)

[4]. Bermond, J.C. Darrot, E. Delmos, Perennes, S., Hamilton Cycle

[5]. Decomposition of the butterfly Network, Parallel rocessing Letters,

[6]. Vol. 8,(1998),371-385

[7]. Chang, G.J., Algorithmic aspects of domination in graphs, in :D.-Z.Du, P.M. Pardalos (Eds.), Hand book of combinational Otimization, Vol. 3, Kluwer, Boston, MA,(1998),pp.339-405.

[8]. Fink, J.F., Jacobson, M.S., Kinch L.F., Roberts, J., The bondage number of graph,Descrete Math. Vol.86(1990) ,47-57.

[9]. Haynes, T.W., Hedetniemi, S.T., Slater, P.J., Fundamentals of Domination in Graphs: Advanced topics, Marcel Dekker, New York,(1998)

[10]. Kelkar, I.P., Some Studies On Domination Paramaters of Butterfly Graphs, Ph.D Thesis, Sri Padmavati Mahila Visvavidyalayam Tirupati India,(2006)

[11]. Leighton F.T., Introduction to Parallel Algorithms and Architecture Arrays, Trees, Hypercudes,Morgan Kaufman,San Mateo,CA (1992).

[12]. Ore, O., Theory of Graphs, Amer, Maths. Soc. Colloq. Pub. 38, Providence (1962) 